\title{
Polygons Whose Vertex Triangles \\ Have Equal Area
}

\section{Guershon Harel and Jeffrey M. Rabin}

1. INTRODUCTION. Consider an $N$-sided polygon $P_{1} P_{2} \cdots P_{N}$ in the Euclidean plane. We denote by $\mathbf{P}_{i}$ the position vector of vertex $P_{i}$ relative to some fixed origin. The (oriented) sides of the polygon are then described by the vectors $\mathbf{s}_{i}=\mathbf{P}_{i+1}-\mathbf{P}_{i}$. Here and throughout this paper we extend the labeling of vertices and sides cyclically when any index falls outside the range $1 \leq i \leq N$, so that $\mathbf{P}_{0}=\mathbf{P}_{N}$ and $\mathbf{s}_{i}=\mathbf{s}_{i+k N}$ for any integers $i$ and $k$. Each triple of consecutive vertices $P_{i-1}, P_{i}, P_{i+1}$ defines the vertex triangle $P_{i-1} P_{i} P_{i+1}$ of the vertex $P_{i}$; its sides are $\mathbf{s}_{i-1}, \mathbf{s}_{i}$, and the diagonal $\mathbf{P}_{i-1}-\mathbf{P}_{i+1}$. We ask: For which polygons do all vertex triangles have the same (nonzero) area?

Definition 1. A polygon $P_{1} P_{2} \cdots P_{N}$ for which all vertex triangles $P_{i-1} P_{i} P_{i+1}$ have the same nonzero area is called an equal-area polygon.

Clearly every regular polygon has the equal-area property. Our interest in this problem began with the search for a converse to this statement. It is certainly not true that every equal-area polygon is regular. Since a nonsingular linear transformation of the plane multiplies all areas by a common nonzero factor, it preserves the equal-area property. Since translations also do so (trivially), equal-area polygons fall into orbits under the action of the affine group of the plane. In particular, the affine image of a regular polygon is equal-area. These affine regular polygons often appear in geometric problems with affine invariance (see [1], [2], [3], [4], [5], or [6]).

It is not even true that every equal-area polygon is affine regular, as can be seen by counting parameters. The $N$ vertices of an equal-area $N$-gon represent $2 N$ real parameters. Equality of the areas of the $N$ vertex triangles imposes $N-1$ algebraic conditions when $N \geq 5$ (for $N=4$ these conditions are not all independent). Since the affine group can fix six parameters, we expect to find a $2 N-(N-1)-6=N-5$ parameter family of affine equivalence classes of equal-area $N$-gons for $N \geq 5$. Our goal in this paper is to make this parametrization explicit.

We are aware of only two previous discussions of this problem in the literature, both restricted to the case of a convex pentagon ([7] and [8]). These papers pose the problem: If the common area of the vertex triangles is unity, is the area of the pentagon determined? In answering this question affirmatively, they determine all convex equalarea pentagons, but do not recognize them as the affine images of a regular pentagon. The problem is attributed to Robert Davis of Rutgers University, now deceased. One of us (Harel) learned of it from the author of [8].

Consider the first nontrivial instance of the equal-area problem, the quadrilateral shown in Figure 1. Vertex triangles $P_{1} P_{2} P_{3}$ and $P_{2} P_{3} P_{4}$ have common base $P_{2} P_{3}$, so their areas are equal if and only if their heights are equal, which is true precisely when sides $P_{1} P_{4}$ and $P_{2} P_{3}$ are parallel. Applying the same reasoning to the other vertex triangles, we conclude that $P_{1} P_{2} P_{3} P_{4}$ must be a parallelogram. We recognize this as the affine image of a regular 4-gon (square).

However, if we do not insist that an equal-area quadrilateral be convex (a convex polygon being one that bounds a convex region), or even simple (meaning that all 


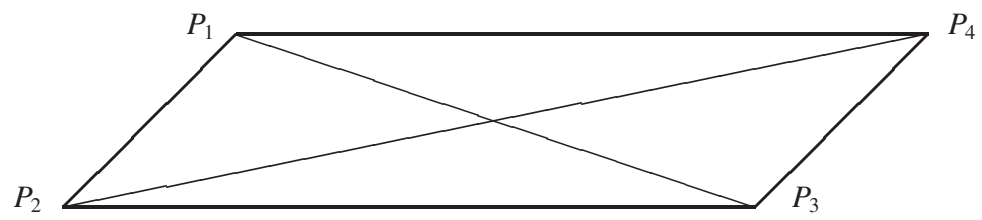

Figure 1. A convex equal-area quadrilateral is a parallelogram.

vertices are distinct and sides may intersect only at vertices that are endpoints of each), there is another possibility, as shown in Figure 2. The segment $P_{1} P_{2}$ is parallel to $P_{3} P_{4}$, and the external diagonals $P_{1} P_{3}$ and $P_{2} P_{4}$ are parallel. Another description of the situation is that an equal-area quadrilateral of this type can be obtained from that in Figure 1 by reflecting vertex $P_{4}$ through vertex $P_{3}$.

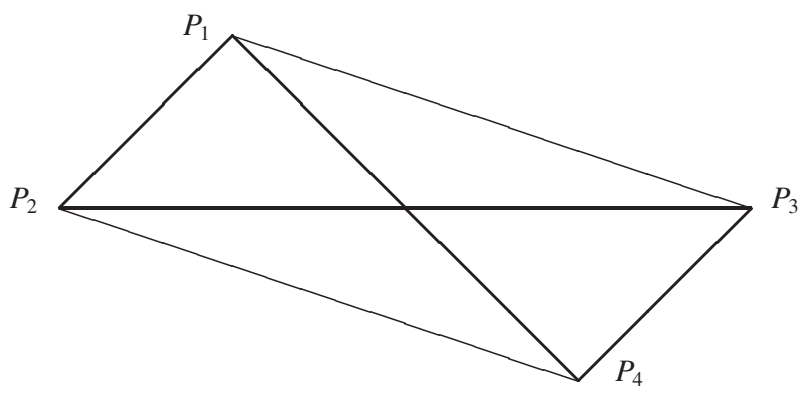

Figure 2. A nonconvex, nonsimple equal-area quadrilateral with external diagonals.

Since the areas of the vertex triangles are nonzero, each consecutive pair of sides $\left\{\mathbf{s}_{i}, \mathbf{s}_{i+1}\right\}$ of an equal-area polygon forms a basis for the plane. The essential difference between Figures 1 and 2 is that in the former case all these bases have the same orientation, whereas in the latter case they do not. For convex polygons these orientations must all agree.

We can define a space of equal-area $N$-gons as a topological subspace of $\mathbb{R}^{2 N}$ by using the $2 N$ coordinates of the vertices $P_{i}$ as the coordinates in $\mathbb{R}^{2 N}$. Alternatively, we can identify polygons related by a translation by using the components of the side vectors $\mathbf{s}_{i}$ as coordinates. It is clear from the quadrilateral case that these spaces have several connected components. One cannot change the orientations of the bases $\left\{\mathbf{s}_{i}, \mathbf{s}_{i+1}\right\}$ by continuously deforming the polygon. That would require the area of some, and therefore every, vertex triangle to pass through zero, which was excluded from the definition of an equal-area polygon precisely in order to obtain a nice decomposition into connected components. In order to include all components, we use the term "polygon" in the most general sense of a closed polygonal curve, and allow nonconvex and nonsimple examples. Our interest is in the space of affine equivalence classes of equalarea polygons. We will introduce discrete parameters $\mu_{i}$ labeling the components, and continuous parameters $x_{i}$ embedding each component in $\mathbb{R}^{N}$ as an algebraic variety, the zero locus of explicit polynomial equations. As we will explain, the polynomials appearing in these equations are closely related to continued fractions.

We invite the reader to continue the geometric reasoning we applied to quadrilaterals to study the cases $N=5$ and $N=6$, at least for convex polygons, and compare with the results we obtain algebraically in what follows. An interesting example of an equal-area octagon (suggested by one of the referees) appears in Figure 3. Four con- 


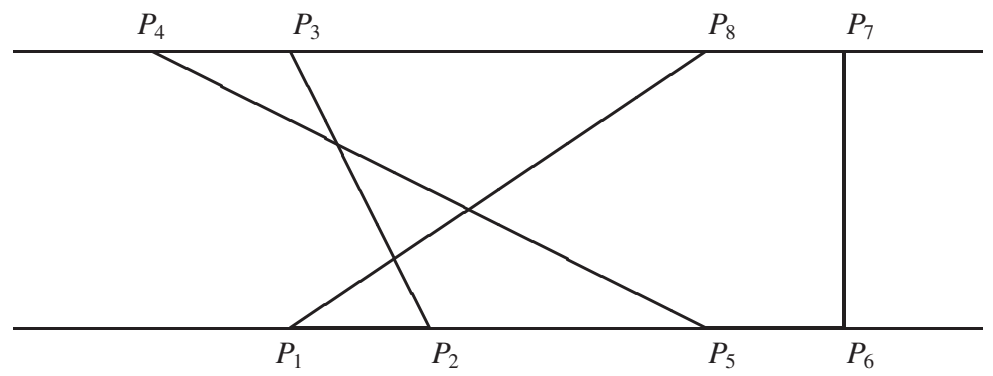

Figure 3. An equal-area octagon.

gruent sides lie on two parallel lines, and pairs of these sides define parallelograms of equal area. Each vertex triangle of the octagon is a vertex triangle of one such parallelogram.

2. BASIC EQUATIONS. Let us now try to formulate the general problem algebraically. Fix a positive orientation of the plane, and attach a sign $\sigma_{i}= \pm 1$ to vertex $P_{i}$ according as the sides $\left\{\mathbf{s}_{i-1}, \mathbf{s}_{i}\right\}$ incident on this vertex form a positively or negatively oriented basis. Considering the plane to be embedded in $\mathbb{R}^{3}$, the vectors $\mathbf{s}_{i-1} \times \mathbf{s}_{i}$ are all normal to the plane, the particular normal direction being determined by $\sigma_{i}$, and of magnitude twice the common area of the vertex triangles. Therefore, the equal-area conditions can be written as

$$
\sigma_{i} \mathbf{s}_{i-1} \times \mathbf{s}_{i}=\sigma_{i+1} \mathbf{s}_{i} \times \mathbf{s}_{i+1},
$$

or

$$
\left(\sigma_{i} \mathbf{s}_{i-1}+\sigma_{i+1} \mathbf{s}_{i+1}\right) \times \mathbf{s}_{i}=0 .
$$

Multiplying through by $\sigma_{i}$ and defining $\mu_{i}=\sigma_{i} \sigma_{i+1}$, we obtain

$$
\left(\mathbf{s}_{i-1}+\mu_{i} \mathbf{s}_{i+1}\right) \times \mathbf{s}_{i}=0 .
$$

The sign $\mu_{i}$ should be viewed as attached to side $\mathbf{s}_{i}$. It tells whether the two bases of consecutive sides containing that side have the same $(+1)$ or the opposite $(-1)$ orientation. This information is independent both of the orientation chosen for the plane and of the orientation for the polygon specified by the cyclic order of its vertices. Intuitively, $\sigma_{i}=+1(-1)$ if in traversing the polygon we turn left (right) at $P_{i}$, while $\mu_{i}=+1(-1)$ if the turns entering and leaving side $\mathbf{s}_{i}$ are in the same (opposite) direction. A convex polygon must have all $\mu_{i}=+1$, but of course $\mu_{i}=+1$ for every $i$ is not sufficient to guarantee convexity. Given the $\mu_{i}$ and an initial orientation $\sigma_{1}$ at $P_{1}$, the remaining $\sigma_{j}$ can be found from

$$
\sigma_{j}=\sigma_{1} \prod_{i=1}^{j-1} \mu_{i} .
$$

Since $\sigma_{N+1}=\sigma_{1}$ this imposes the constraint

$$
\prod_{i=1}^{N} \mu_{i}=1
$$


that is, there must be an even number of orientation changes upon traversing the boundary of the polygon once.

According to equation (1), the vectors $\mathbf{s}_{i-1}+\mu_{i} \mathbf{s}_{i+1}$ and $\mathbf{s}_{i}$ are parallel, so there are real numbers $x_{i}$ such that

$$
\mathbf{s}_{i-1}+\mu_{i} \mathbf{s}_{i+1}+x_{i} \mathbf{s}_{i}=\mathbf{0} .
$$

If $\mu_{i}=+1$, this is equivalent to $\mathbf{P}_{i+2}-\mathbf{P}_{i-1}=\left(1-x_{i}\right) \mathbf{s}_{i}$, which says that the diagonal $\mathbf{P}_{i+2}-\mathbf{P}_{i-1}$ must be parallel to side $\mathbf{s}_{i}$, and $\left(1-x_{i}\right)$ times longer than this side. If $\mu_{i}=-1$, condition (2) becomes $\left(2 \mathbf{P}_{i+1}-\mathbf{P}_{i+2}\right)-\mathbf{P}_{i-1}=\left(1-x_{i}\right) \mathbf{s}_{i}$, which says that the segment joining $P_{i-1}$ to the reflection of $P_{i+2}$ through $P_{i+1}$ must be parallel to and $\left(1-x_{i}\right)$ times longer than $\mathbf{s}_{i}$. These conditions generalize those obtained in the quadrilateral cases. If the three vertices $P_{i-1}, P_{i}$, and $P_{i+1}$ and the numbers $\mu_{i}$ and $x_{i}$ are known, they allow the next vertex $P_{i+2}$ to be located geometrically. Adding the requirement that the polygon with sides $\mathbf{s}_{i}$ be closed, we have the complete set of equations determining an equal-area polygon:

$$
\begin{gathered}
\mathbf{s}_{i-1}+x_{i} \mathbf{s}_{i}+\mu_{i} \mathbf{s}_{i+1}=\mathbf{0}, \\
\mathbf{s}_{1}+\mathbf{s}_{2}+\cdots+\mathbf{s}_{N}=\mathbf{0} .
\end{gathered}
$$

As promised, each given sequence of signs $\mu_{i}, i=1,2, \ldots, N$, labels a connected component (possibly empty) of the space of equal-area $N$-gons. Our analysis will begin with the component having $\mu_{i}=+1$ for all $i$, which contains all convex examples, and consider the other components afterward. The parameters $\mu_{i}$ and $x_{i}$ are constant on each affine orbit of equal-area $N$-gons, and the affine symmetry is explicitly visible in equations (3) and (4), which are invariant under translations (since the $\mathbf{s}_{i}$ are) and linear transformations of the $\mathbf{s}_{i}$.

Given an equal-area polygon satisfying equations (3) and (4), we can by means of an affine transformation fix $P_{1}$ to be the origin and $\left\{\mathbf{s}_{1}, \mathbf{s}_{2}\right\}$ to be the standard basis of the plane. Knowledge of the $\mu_{i}$ and $x_{i}$ then determines successively all the remaining sides. In this sense the numbers $\mu_{i}$ and $x_{i}$ directly parametrize equal-area polygons (with marked vertices) modulo affine equivalence. A cyclic permutation of these parameters corresponds to a relabeling of the vertices of the polygon.

We recast the equal-area and closure conditions of equations (3) and (4) in matrix form by defining

$$
\begin{gathered}
X=\left[\begin{array}{ccccccc}
x_{1} & \mu_{1} & 0 & 0 & \cdots & 0 & 1 \\
1 & x_{2} & \mu_{2} & 0 & \cdots & 0 & 0 \\
0 & 1 & x_{3} & \mu_{3} & \cdots & 0 & 0 \\
\vdots & \vdots & \vdots & \vdots & & \vdots & \vdots \\
\mu_{N} & 0 & 0 & 0 & \cdots & 1 & x_{N}
\end{array}\right], \quad S=\left[\begin{array}{c}
\mathbf{s}_{1} \\
\mathbf{s}_{2} \\
\vdots \\
\mathbf{s}_{N}
\end{array}\right], \\
E=\left[\begin{array}{llll}
1 & 1 & \cdots & 1
\end{array}\right] .
\end{gathered}
$$

That is, $S$ is the $N \times 2$ matrix whose rows are the vectors $\mathbf{s}_{i}$, and $E$ is the $1 \times N$ matrix with each entry equal to unity. We can then summarize the discussion in this section in a theorem.

Theorem 1. There exists an equal-area polygon having sides $\mathbf{s}_{i}$ and parameters $\mu_{i}$ and $x_{i}$ if and only if

$$
X S=0, \quad E S=0,
$$


and $S$ has rank 2. Two equal-area polygons are affinely equivalent, preserving the labeling of vertices, if and only if they have the same data $X$.

3. SYMMETRIC EQUAL-AREA POLYGONS. In this section we prove an appropriate converse to our initial observation that regular polygons have the equal-area property.

Definition 2. We call an equal-area polygon symmetric if all $\mu_{i}=+1$ and all $x_{i}$ have a common value $x$.

This class includes the affine regular polygons mentioned earlier. For a symmetric equal-area polygon, (5) becomes

$$
C S=-x S, \quad E S=0,
$$

where $C$ is the circulant matrix

$$
C=\left[\begin{array}{ccccccc}
0 & 1 & 0 & 0 & \cdots & 0 & 1 \\
1 & 0 & 1 & 0 & \cdots & 0 & 0 \\
0 & 1 & 0 & 1 & \cdots & 0 & 0 \\
\vdots & \vdots & \vdots & \vdots & & \vdots & \vdots \\
0 & 0 & 0 & 0 & \cdots & 0 & 1 \\
1 & 0 & 0 & 0 & \cdots & 1 & 0
\end{array}\right]
$$

Thus, the possible values of $-x$ are the eigenvalues of $C$. Since the $N \times 2$ matrix $S$ must have rank 2, its columns, which are eigenvectors of $C$, must be linearly independent. The acceptable eigenvalues therefore have geometric multiplicity at least two.

It is easy to diagonalize any circulant matrix [4]. Begin with the elementary circulant matrix $\Pi$ having 1s on the subdiagonal (and upper right corner) and all other entries zero. As a linear transformation $\Pi$ is a right cyclic shift of the components of a vector. This makes it clear that its eigenvalues are $\lambda_{k}=w^{k}$ for $k=1, \ldots, N$, with corresponding eigenvectors $\mathbf{v}_{k}=\left[w^{k}, w^{2 k}, \ldots, w^{(N-1) k}, 1\right]^{T}$, where $w=\exp (2 \pi i / N)$ is the fundamental $N$ th root of unity. Since $C=\Pi+\Pi^{-1}$, it has the same eigenvectors $\mathbf{v}_{k}$, with eigenvalues $w^{k}+w^{-k}=2 \cos (2 k \pi / N)$. Because these eigenvalues are real, the real and imaginary parts of the eigenvectors are again eigenvectors, and the eigenspaces are two-dimensional unless $\mathbf{v}_{k}$ is already real. Consistent with this, the eigenvalues corresponding to $k$ and $N-k$ are actually equal.

The distinct eigenvalues having multiplicity two are $2 \cos (2 k \pi / N)$ for $k=1,2$, $\ldots,[(N-1) / 2]$. The eigenvectors give the columns of $S$, and then the rows are

$$
\mathbf{s}_{j}=\left(\cos \frac{2 j k \pi}{N}, \sin \frac{2 j k \pi}{N}\right)
$$

Observe that the closure condition for an equal-area polygon, $E S=0$, is automatically satisfied for these solutions, as a consequence of the fact that

$$
1+w^{k}+w^{2 k}+\cdots+w^{(N-1) k}=\frac{w^{N k}-1}{w^{k}-1}=0
$$

when $0<k<N$. 
The solutions obtained have an obvious geometric description. Mark off the $N$ th roots of unity on the unit circle and choose one as the initial vertex $P_{1}$. For $k=1$, take successive vertices to be the successive roots in cyclic order, resulting in a regular polygon. For $k>1$, take successive vertices to be the roots $k$ steps later in cyclic order, resulting in a generalized regular polygon [3]. If $k$ and $N$ are relatively prime, this will be a nonconvex star $N$-gon; otherwise it will be an $(N / d)$-gon traversed $d$ times, where $d=\operatorname{gcd}(k, N)$. Recalling the affine group action we obtain our second major result:

Theorem 2. Every symmetric equal-area polygon is a generalized affine regular polygon.

Such a polygon has both a circumscribed and an inscribed ellipse, the affine images of the circumscribed and inscribed circles of a regular (star) polygon.

4. NONSYMMETRIC EQUAL-AREA POLYGONS. We return to the analysis of equations (5), assuming now only that the relative orientations $\mu_{i}=+1$ for every $i$. This can be viewed as a generalized eigenvalue problem in which the $x_{i}$ must be determined so that the null space of the matrix $X$ contains two linearly independent vectors that can serve as the columns of $S$. (This is distantly related to the problem of determining the periodic Jacobi matrix $X$ from its spectral data [9], [10].) The closure condition $E S=0$ will now be a nontrivial constraint in general.

Introducing the notation $X^{(i)}$ for the $i$ th row of $X$, we see by inspection that $X^{(2)}, X^{(3)}, \ldots, X^{(N-1)}$ are linearly independent. Since we require the rank of $X$ to be $N-2$, these $N-2$ vectors must span the row space, and we can find a basis for the null space by explicitly constructing a pair of linearly independent vectors orthogonal to them. Indeed, the first (or last) two components of a vector in the null space can be chosen arbitrarily, and then each successive component is determined by orthogonality to $X^{(2)}, X^{(3)}, \ldots$, in that order (or the reverse). These components will be given by polynomials in the $x_{i}$.

To make this explicit, we introduce the notation $\left|x_{i}, x_{j}\right|$, where $i \leq j$, for the polynomial in $x_{i}, x_{i+1}, \ldots, x_{j}$ given by the tridiagonal determinant

$$
\left|x_{i}, x_{j}\right|=\left|\begin{array}{ccccc}
x_{i} & 1 & \cdots & 0 & 0 \\
1 & x_{i+1} & \cdots & 0 & 0 \\
\vdots & \vdots & \ddots & \vdots & \vdots \\
0 & 0 & \cdots & x_{j-1} & 1 \\
0 & 0 & \cdots & 1 & x_{j}
\end{array}\right| .
$$

This notation is invariant under simultaneous, but not separate shifts of $i$ and $j$ by $N$. Cofactor expansion shows that these polynomials obey the recursion relations

$$
\begin{aligned}
\left|x_{i}, x_{j}\right| & =x_{i}\left|x_{i+1}, x_{j}\right|-\left|x_{i+2}, x_{j}\right| \\
& =\left|x_{i}, x_{j-1}\right| x_{j}-\left|x_{i}, x_{j-2}\right| .
\end{aligned}
$$

Sample values are $\left|x_{1}, x_{1}\right|=x_{1},\left|x_{1}, x_{2}\right|=x_{1} x_{2}-1$, and $\left|x_{1}, x_{3}\right|=x_{1} x_{2} x_{3}-x_{1}-x_{3}$. One can extend the definition to some cases where $i>j$ in a manner consistent with the recursion relations by setting $\left|x_{i}, x_{i-1}\right|=1,\left|x_{i}, x_{i-2}\right|=0$, and $\left|x_{i}, x_{i-3}\right|=-1$.

Relations (7) guarantee that the following four vectors are orthogonal to each of $X^{(2)}, X^{(3)}, \ldots, X^{(N-1)}$ and therefore belong to the null space: 


$$
\begin{aligned}
& \mathbf{n}_{1}^{+}=\left[-\left|x_{3}, x_{0}\right|,+\left|x_{3}, x_{1}\right|,-\left|x_{3}, x_{2}\right|, \ldots,(-1)^{N}\left|x_{3}, x_{N-1}\right|\right]^{T}, \\
& \mathbf{n}_{2}^{+}=\left[-\left|x_{2}, x_{0}\right|,+\left|x_{2}, x_{1}\right|,-\left|x_{2}, x_{2}\right|, \ldots,(-1)^{N}\left|x_{2}, x_{N-1}\right|\right]^{T}, \\
& \mathbf{n}_{1}^{-}=\left[(-1)^{N}\left|x_{2}, x_{N-2}\right|,(-1)^{N-1}\left|x_{3}, x_{N-2}\right|, \ldots,-\left|x_{N+1}, x_{N-2}\right|\right]^{T}, \\
& \mathbf{n}_{2}^{-}=\left[(-1)^{N}\left|x_{2}, x_{N-1}\right|,(-1)^{N-1}\left|x_{3}, x_{N-1}\right|, \ldots,-\left|x_{N+1}, x_{N-1}\right|\right]^{T} .
\end{aligned}
$$

As a basis for the null space one can take either $\left\{\mathbf{n}_{1}^{+}, \mathbf{n}_{2}^{+}\right\}$or $\left\{\mathbf{n}_{1}^{-}, \mathbf{n}_{2}^{-}\right\}$. Note that we have exploited the freedom to choose the first or last two components of a null space vector by taking $\mathbf{n}_{1}^{+}=[1,0, \ldots]^{T}, \mathbf{n}_{2}^{+}=[0,1, \ldots]^{T}$, and $\mathbf{n}_{1}^{-}=[\ldots, 0,1]^{T}$, $\mathbf{n}_{2}^{-}=[\ldots, 1,0]^{T}$.

We mention that the expressions $\left|x_{1}, x_{N}\right|$ have a long history in number theory [11], [12]: the numerators and denominators of continued fractions are given by

$$
x_{1}-\frac{1 \mid}{\mid x_{2}}-\frac{1 \mid}{\mid x_{3}}-\cdots-\frac{1 \mid}{\mid x_{N}}=\frac{\left|x_{1}, x_{N}\right|}{\left|x_{2}, x_{N}\right|} .
$$

The recursion relations, and many other identities, were known to Euler in this context. The determinant form (6) is due to Sylvester. The following description of $\left|x_{1}, x_{N}\right|$ is easily proved by induction: $\left|x_{1}, x_{N}\right|$ is the sum of $x_{1} x_{2} \cdots x_{N}$ and all monomials that can be obtained from it by deleting pairs of consecutive variables $x_{i} x_{i+1}$, changing the sign with each deletion. (The empty monomial is interpreted as unity.) Note that linear rather than cyclic order is used here: $x_{N} x_{1}$ is not considered a pair of consecutive variables. In the symmetric case $x_{i}=x$ for all $i,\left|x_{1}, x_{N}\right|$ is equal to $U_{N}(x / 2)$, where the $U_{N}$ are the Chebyshev polynomials of type II, which can be defined by the generating function

$$
\frac{1}{1-2 x t+t^{2}}=\sum_{N=0}^{\infty} U_{N}(x) t^{N} .
$$

In fact, a connection between continued fractions and the equal-area problem is not surprising. See [13] for a geometric treatment of continued fractions as slopes of vectors $\mathbf{s}_{i}$ obeying the recursion relation $\mathbf{s}_{i+1}=\mathbf{s}_{i-1}+x_{i} \mathbf{s}_{i}$. This is equation (3) with all $\mu_{i}=-1$, leading to continued fractions (8) with all minus signs changed to plus (see section 5). The equal-area property plays a central role in this treatment.

At this point we can express the conditions on the parameters $x_{i}$ that produce an equal-area polygon. In order for $X$ to have rank $N-2, X^{(1)}$ and $X^{(N)}$ must be in the span of the other rows, which is to say that they must be orthogonal to the null space bases we have determined. We express this by the four equations

$$
X^{(1)} \cdot \mathbf{n}_{1}^{+}=X^{(1)} \cdot \mathbf{n}_{2}^{+}=X^{(N)} \cdot \mathbf{n}_{1}^{-}=X^{(N)} \cdot \mathbf{n}_{2}^{-}=0 .
$$

(Of course, one could use a common basis $\left\{\mathbf{n}_{1}^{+}, \mathbf{n}_{2}^{+}\right\}$for all four equations, but our choice leads to some simplification and an increase in symmetry.) We supplement this with the closure constraint $E S=0$ in the form of two additional equations $E \cdot \mathbf{n}_{1}^{+}=$ $E \cdot \mathbf{n}_{2}^{+}=0$. This produces the following six equations, five of which are independent:

$$
\begin{gathered}
x_{1}+(-1)^{N}\left|x_{3}, x_{N-1}\right|=0, \\
1+(-1)^{N}\left|x_{2}, x_{N-1}\right|=0, \\
x_{N}+(-1)^{N}\left|x_{2}, x_{N-2}\right|=0,
\end{gathered}
$$




$$
\begin{gathered}
1+(-1)^{N}\left|x_{2}, x_{N-1}\right|=0 \\
\sum_{i=4}^{N}(-1)^{i}\left|x_{3}, x_{i-1}\right|=0 \\
1+\sum_{i=3}^{N}(-1)^{i}\left|x_{2}, x_{i-1}\right|=0 .
\end{gathered}
$$

Equations (9) and (11) express $x_{1}$ and $x_{N}$ explicitly in terms of the remaining variables, so we obtain:

Theorem 3. There exists an equal-area polygon with each $\mu_{i}=+1$ and parameters $x_{2}, x_{3}, \ldots, x_{N-1}$ if and only if the following equations hold:

$$
\begin{gathered}
1+(-1)^{N}\left|x_{2}, x_{N-1}\right|=0 \\
\sum_{i=4}^{N}(-1)^{i}\left|x_{3}, x_{i-1}\right|=0 \\
1+\sum_{i=3}^{N}(-1)^{i}\left|x_{2}, x_{i-1}\right|=0
\end{gathered}
$$

In this event $x_{1}$ and $x_{N}$ are given explicitly by equations (9) and (11), respectively.

Given a solution to these equations, a set of sides of a corresponding equal-area polygon is given by $S=\left[\begin{array}{ll}\mathbf{n}_{1}^{+} & \mathbf{n}_{2}^{+}\end{array}\right]$:

$$
\mathbf{s}_{i}=(-1)^{i}\left(\left|x_{3}, x_{i-1}\right|,\left|x_{2}, x_{i-1}\right|\right)
$$

In particular, $\mathbf{s}_{1}=(1,0)$ and $\mathbf{s}_{2}=(0,1)$, so this choice fixes the common area of the vertex triangles to be $1 / 2$. As noted earlier, the slopes of the vectors $\mathbf{s}_{i}$ are given by continued fractions in the $x_{i}$.

For example, in the case $N=5$, the equations to be solved are

$$
x_{2} x_{3} x_{4}-x_{2}-x_{4}=1, \quad x_{3} x_{4}-x_{3}=1, \quad x_{2} x_{3}-x_{2}=1 .
$$

It is not difficult to deduce from these equations that $x_{2}=x_{3}=x_{4}$, and that the only equal-area pentagons (with $\mu_{i}=+1$ ) are the symmetric ones found in the previous section.

In the general case with $N>5$, we will solve the constraints for $x_{2}, x_{3}$, and $x_{4}$ in terms of $x_{5}, x_{6}, \ldots, x_{N-1}$, thereby determining the part of the variety of equal-area $N$-gons on which the latter variables can be used as local coordinates. This can be done by using the first recursion relation in (7) to make explicit the dependence on $x_{2}, x_{3}$, and $x_{4}$ in equations (10), (12), and (13). The equations become, after some simplification,

$$
\begin{gathered}
\alpha\left(x_{2} x_{3} x_{4}-x_{2}-x_{4}\right)-\beta\left(x_{2} x_{3}-1\right)=1, \\
A\left(x_{3} x_{4}-1\right)-B x_{3}=0, \\
x_{2}=A x_{4}-B+1
\end{gathered}
$$


where

$$
\begin{aligned}
& \alpha=(-1)^{N-1}\left|x_{5}, x_{N-1}\right|, \\
& \beta=(-1)^{N-1}\left|x_{6}, x_{N-1}\right|, \\
& A=1+\sum_{i=5}^{N-1}(-1)^{i}\left|x_{5}, x_{i}\right|, \\
& B=1+\sum_{i=5}^{N-1}(-1)^{i}\left|x_{6}, x_{i}\right| .
\end{aligned}
$$

Elimination of $x_{2}$ and $x_{4}$ results in the quadratic in $x_{3}$,

$$
(\alpha B-\beta A) x_{3}^{2}+[(A-1)(\alpha B-\beta A)-A] x_{3}-\alpha A=0 .
$$

If the discriminant of this quadratic is positive in some region of the $\left(x_{5}, x_{6}, \ldots, x_{N-1}\right)$ hyperplane in $\mathbb{R}^{N}$, we get two real solutions for $x_{3}$ there. Provided that $A x_{3} \neq 0, x_{2}$ and $x_{4}$ are then uniquely determined by $x_{3}$, so over this region the variety of equal-area polygons has two branches and is a smooth manifold of dimension $N-5$, as expected. The branches meet at the discriminant locus of the quadratic, where $x_{5}, x_{6}, \ldots, x_{N-1}$ are no longer good local coordinates. The existence of such a region and some information about its extent are furnished by

Theorem 4. Fix nonzero constants $t_{i}$ for $i=5,6, \ldots, N-1$ and consider the line $x_{i}=t_{i} x$ parametrized by the real variable $x$ in the $\left(x_{5}, x_{6}, \ldots, x_{N-1}\right)$-hyperplane. For sufficiently large $|x|$, the discriminant of (14) restricted to this line is positive, and $A x_{3} \neq 0$.

Proof. We show that after substituting $x_{i}=t_{i} x$ in the discriminant $b^{2}-4 a c$ of (14), the $b^{2}$ term has higher degree in $x$ than the $4 a c$ term. The parameters $\alpha$ and $A$ have degree $N-5$ by definition, but the degree of

$$
\begin{aligned}
\alpha B-\beta A= & (-1)^{N-1}\left|x_{5}, x_{N-1}\right| \\
& +\sum_{i=4}^{N-1}(-1)^{N+i-1}\left(\left|x_{5}, x_{N-1}\right|\left|x_{6}, x_{i}\right|-\left|x_{6}, x_{N-1}\right|\left|x_{5}, x_{i}\right|\right)
\end{aligned}
$$

is less clear because of numerous cancellations in the sum. This sum can be simplified with the help of the identity,

$$
\left|x_{i}, x_{j}\right|\left|x_{k}, x_{l}\right|-\left|x_{k}, x_{j}\right|\left|x_{i}, x_{l}\right|=\left|x_{i+1}, x_{j}\right|\left|x_{k+1}, x_{l}\right|-\left|x_{k+1}, x_{j}\right|\left|x_{i+1}, x_{l}\right|,
$$

which follows easily from the recursion relations (7). Using (15) recursively in the expression for $\alpha B-\beta A$ to replace the pair $x_{5}, x_{6}$ by $x_{i+1}, x_{i+2}$ shows that

$$
\alpha B-\beta A=\sum_{i=5}^{N-1}(-1)^{N+i}\left|x_{i}, x_{N-1}\right|,
$$

which has degree $N-5$. The square of the coefficient of $x_{3}$ in the discriminant therefore has degree $4(N-5)$, while the product of the leading and constant coefficients 
has degree $3(N-5)$. Since $N>5$, the discriminant is positive for large $|x|$ as claimed. Furthermore, $\alpha A$ must be large for large $|x|$, so $x_{3}=0$ cannot be a root of (14) in this region, whence $A x_{3} \neq 0$.

It is natural to conjecture on this basis that the region where the discriminant is nonpositive is compact, but unfortunately this conjecture is false, as shown by the following example. Set $N=14$, and assume that $x_{5}, x_{6}, x_{8}, x_{9}, x_{12}$, and $x_{13}$ all vanish. Then it is easy to check that $A=1$, which kills the "dominant" term in the coefficient of $x_{3}$. In fact, the discriminant is

$$
1-4 x_{7} x_{10}^{2} x_{11}^{2}+4 x_{7} x_{10} x_{11}+4 x_{10} x_{11}^{2} .
$$

For arbitrarily large $x_{10}$ and $x_{11}$, the expression (16) can be either positive or negative.

As an example of these results we discuss the case $N=6$. We find that

$$
\alpha=-x_{5}, \quad \beta=-1, \quad A=1-x_{5}, \quad B=0 .
$$

The fact that $B=0$ leads to a great simplification: the quadratic (14) reduces to

$$
\left(1-x_{5}\right)\left(x_{3}-1\right)\left(x_{3}-x_{5}\right)=0 \text {. }
$$

If $x_{5}=1, x_{3}$ is undetermined by this equation. This is also the discriminant locus for the quadratic, and at this point the coordinate $x_{5}$ on the space of equal-area hexagons should be replaced by $x_{3}$. Solving the quadratic, the first branch $x_{3}=1$ leads to $x_{1}=$ $x_{4}=x_{6}=1, x_{2}=2-x_{5}$. The sides of the equal-area hexagons on this branch are given by:

$$
\begin{aligned}
& \mathbf{s}_{1}=(1,0), \quad \mathbf{s}_{2}=(0,1), \quad \mathbf{s}_{3}=\left(-1, x_{5}-2\right), \\
& \mathbf{s}_{4}=\left(1,1-x_{5}\right), \quad \mathbf{s}_{5}=(0,1), \quad \mathbf{s}_{6}=(-1,-1) .
\end{aligned}
$$

A typical hexagon in this family is shown in Figure 4. All vertices are fixed except $P_{4}$, which moves vertically as $x_{5}$ varies. It goes to infinity as $x_{5}$ does. At $x_{5}=1$ we obtain the degenerate symmetric case of a triangle traversed twice.

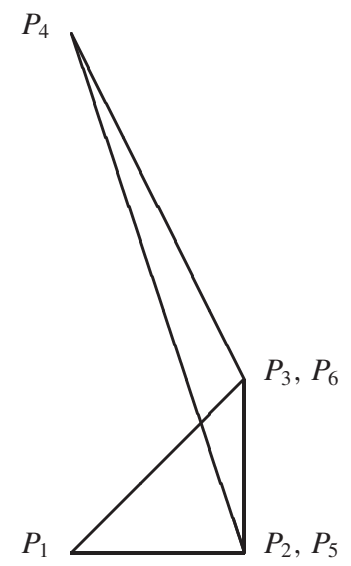

Figure 4. An equal-area hexagon on the first branch. All vertices are fixed except $P_{4}$, which moves vertically with $x_{5}$. 
The second branch $x_{3}=x_{5}$ leads to $x_{1}=x_{3}=x_{5}, x_{2}=x_{4}=x_{6}=1 / x_{5}$, with sides

$$
\begin{array}{llr}
\mathbf{s}_{1}=(1,0), & \mathbf{s}_{2}=(0,1), & \mathbf{s}_{3}=\left(-1,-1 / x_{5}\right), \\
\mathbf{s}_{4}=\left(x_{5}, 0\right), & \mathbf{s}_{5}=\left(0,1 / x_{5}\right) & \mathbf{s}_{6}=\left(-x_{5},-1\right) .
\end{array}
$$

Examples of these equal-area hexagons are shown in Figure 5. Setting $x_{5}=1$ again produces the triangle, while $x_{5}=-1$ leads to the affine image of a regular hexagon. Observe that opposite sides of these hexagons are parallel, because the equal-area condition requires them to be parallel to a common diagonal. (This is not the case on the first branch, because some of the "diagonals" in that setting have zero length.) One can also check that each pair of opposite sides has the same ratio of longer side to shorter side. As $x_{5} \rightarrow \pm \infty$, one side of the polygon becomes short and moves out to infinity. The adjacent sides become long and nearly parallel. This applies as well to the degeneration as $x_{5} \rightarrow 0^{ \pm}$due to the symmetry in the set of $x_{i}$ under the transformation $x_{5} \rightarrow 1 / x_{5}$.
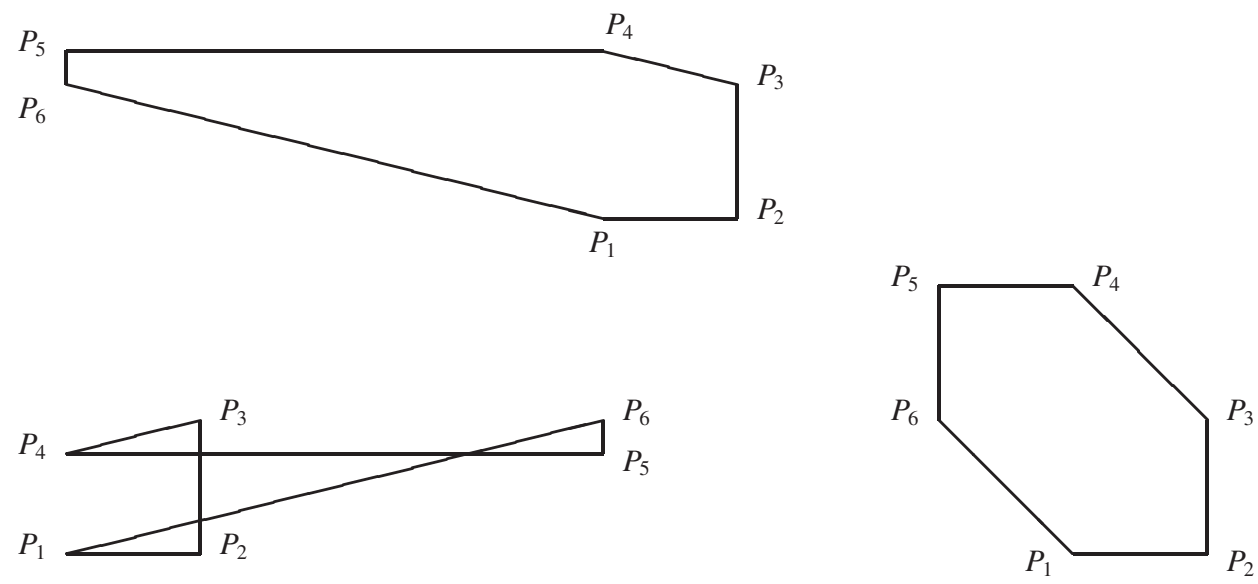

Figure 5. Equal-area hexagons on the second branch. Top left: $x_{5}=-4$. Bottom left: $x_{5}=+4$. Right: $x_{5}=-1$ (affine regular). Vertices $P_{1}, P_{2}$, and $P_{3}$ are fixed in this family; $P_{5}$ and $P_{6}$ go to infinity as $x_{5}$ does.

5. OTHER ORIENTATION CLASSES. Finally we consider equal-area polygons with arbitrary relative orientations $\mu_{i}$. The analysis is very similar to that of the preceding section, with appropriate sign changes. However, we have not made a detailed study of examples or the discriminant in every orientation class.

We define polynomials analogous to the $\left|x_{i}, x_{j}\right|$ via the determinants

$$
\left|x_{i}, x_{j}\right|_{\mu}=\left|\begin{array}{cccccc}
x_{i} & \mu_{i} & 0 & \cdots & 0 & 0 \\
1 & x_{i+1} & \mu_{i+1} & \cdots & 0 & 0 \\
\vdots & \vdots & \vdots & & \vdots & \vdots \\
0 & 0 & 0 & \cdots & x_{j-1} & \mu_{j-1} \\
0 & 0 & 0 & \cdots & 1 & x_{j}
\end{array}\right| .
$$

They obey the recursion relations

$$
\begin{aligned}
\left|x_{i}, x_{j}\right|_{\mu} & =x_{i}\left|x_{i+1}, x_{j}\right|_{\mu}-\mu_{i}\left|x_{i+2}, x_{j}\right|_{\mu} \\
& =\left|x_{i}, x_{j-1}\right|_{\mu} x_{j}-\left|x_{i}, x_{j-2}\right|_{\mu} \mu_{j-1},
\end{aligned}
$$


and $\left|x_{1}, x_{N}\right|_{\mu}$ is related to the continued fraction

$$
x_{1}-\frac{\mu_{1} \mid}{\mid x_{2}}-\frac{\mu_{2} \mid}{\mid x_{3}}-\cdots-\frac{\mu_{N-1} \mid}{\mid x_{N}} .
$$

Clearly $\left|x_{1}, x_{N}\right|_{\mu}$ can be obtained from $\left|x_{1}, x_{N}\right|$ by changing the signs of certain monomials. By multiplying columns and rows of the defining determinant by the $\mu_{i}$ appropriately, we can obtain the following explicit relationship: $\left|x_{1}, x_{N}\right|_{\mu}$ is obtained from $\left|x_{1}, x_{N}\right|$ by replacing each variable $x_{j}$ for $j>1$ with $\mu_{1} \mu_{2} \cdots \mu_{j-1} x_{j}$ and then fixing the overall sign so that the highest-degree term is $+x_{1} x_{2} \cdots x_{N}$. If we arbitrarily set $\sigma_{1}=1$, the change of variables is simply $x_{j} \mapsto \sigma_{j} x_{j}$.

The first recursion relation in (17) shows that the $\left|x_{i}, x_{j}\right|_{\mu}$ can be used to construct the vectors analogous to $\mathbf{n}_{1}^{-}, \mathbf{n}_{2}^{-}$that span the null space of the matrix $X$. On the other hand, the vectors analogous to $\mathbf{n}_{1}^{+}, \mathbf{n}_{2}^{+}$now belong to the left null space. To construct such vectors spanning the null space, we use the modified polynomials

$$
\left|x_{i}, x_{j}\right|_{\mu}^{+}=\left|\begin{array}{cccccc}
\mu_{i} x_{i} & \mu_{i+1} & 0 & \cdots & 0 & 0 \\
1 & \mu_{i+1} x_{i+1} & \mu_{i+2} & \cdots & 0 & 0 \\
\vdots & \vdots & \vdots & & \vdots & \vdots \\
0 & 0 & 0 & \cdots & \mu_{j-1} x_{j-1} & \mu_{j} \\
0 & 0 & 0 & \cdots & 1 & \mu_{j} x_{j}
\end{array}\right| \text {, }
$$

which satisfy the necessary recursion relation

$$
\left|x_{i}, x_{j}\right|_{\mu}^{+} \mu_{j}=\left|x_{i}, x_{j-1}\right|_{\mu}^{+} x_{j}-\left|x_{i}, x_{j-2}\right|_{\mu}^{+} .
$$

By multiplying each column of the defining determinant by the $\mu_{k}$ that appears in that column, we obtain the transpose of the determinant defining $\left|x_{i}, x_{j}\right|_{\mu}$, demonstrating that

$$
\left|x_{i}, x_{j}\right|_{\mu}^{+}=\mu_{i} \mu_{i+1} \cdots \mu_{j}\left|x_{i}, x_{j}\right|_{\mu}=\sigma_{i} \sigma_{j+1}\left|x_{i}, x_{j}\right|_{\mu} .
$$

The equations for an equal-area polygon are then:

$$
\begin{gathered}
\mu_{2} x_{1}+(-1)^{N}\left|x_{3}, x_{N-1}\right|_{\mu}^{+}=0, \\
\mu_{1}+(-1)^{N}\left|x_{2}, x_{N-1}\right|_{\mu}^{+}=0, \\
\mu_{N-1} x_{N}+(-1)^{N} \mu_{N}\left|x_{2}, x_{N-2}\right|_{\mu}=0, \\
1+(-1)^{N} \mu_{N}\left|x_{2}, x_{N-1}\right|_{\mu}=0, \\
\mu_{2}-1+\sum_{i=4}^{N}(-1)^{i}\left|x_{3}, x_{i-1}\right|_{\mu}^{+}=0, \\
1+\sum_{i=3}^{N}(-1)^{i}\left|x_{2}, x_{i-1}\right|_{\mu}^{+}=0 .
\end{gathered}
$$

As before, equations (19) and (21) are not independent, and equations (18) and (20) can be solved for $x_{1}$ and $x_{N}$, leaving three nontrivial equations for the remaining variables.

Figure 6 shows a family of equal-area hexagons in the orientation class $\sigma_{1}, \ldots, \sigma_{6}=$ $+1,+1,+1,-1,-1,-1$, or $\mu_{1}, \ldots, \mu_{6}=+1,+1,-1,+1,+1,-1$. It is obtained by reflection from the family in Figure 5. Those hexagons can be cut along a vertical diagonal into a pair of trapezoids, and reflecting one of these trapezoids across the 


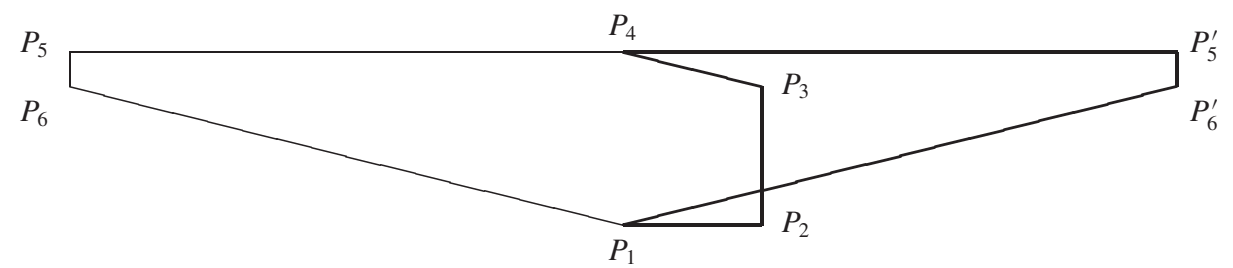

Figure 6. An equal-area hexagon $P_{1} P_{2} P_{3} P_{4} P_{5}^{\prime} P_{6}^{\prime}$ with $\mu_{1}, \ldots, \mu_{6}=+1,+1,-1,+1,+1,-1$ is formed from a hexagon $P_{1} P_{2} P_{3} P_{4} P_{5} P_{6}$ of Figure 5 by reflecting the trapezoid $P_{1} P_{4} P_{5} P_{6}$ across the diagonal $P_{1} P_{4}$.

diagonal gives rise to the new family. Each vertex triangle in the new hexagon is either congruent to one in the original hexagon or has the same base and height. This clearly depends on the high degree of symmetry of the hexagons of Figure 5. It is not obvious how to generalize it to the case $N>6$ or even to $N=6$ with other $\mu_{i}$, although we suspect that some construction based on reflections does apply more generally.

6. CONCLUSION AND OPEN PROBLEMS. We have given explicit equations for the connected components of the variety of affine classes of $N$-gons whose vertex triangles have equal area. We have shown that part of the component having each $\mu_{i}=$ +1 is a smooth manifold on which $x_{5}, x_{6}, \ldots, x_{N-1}$ can be used as local coordinates. And we have given some examples of these polygons, noting interesting degenerations that can occur.

The most interesting open problem would seem to be determining all the singular points of the variety of equal-area $N$-gons and the ways in which the polygons degenerate as these points are approached. In our examples we find that some pairs of sides become parallel and infinitely long, with the sides between them moving out to infinity. One might have expected to observe degenerations in which some sides shrink to zero length, so that the areas of the vertex triangles tend to zero. In fact this is an alternative description of the same type of degeneration. It can be obtained from the previous description by applying affine transformations to keep the polygon bounded as it degenerates. Recall that our choice of the basis $\left\{\mathbf{n}_{1}^{+}, \mathbf{n}_{2}^{+}\right\}$fixes the common area of all vertex triangles to be $1 / 2$, which is why degenerations to zero area are not seen.

It seems to us that there should exist discrete transformations of one equal-area polygon into another, changing either the relative orientations $\mu_{i}$ or the number of sides, but we have not succeeded in finding them. The simple relation between $\left|x_{i}, x_{j}\right|$ and $\left|x_{i}, x_{j}\right|_{\mu}$ suggests that some sequence of reflections in diagonals, sides, or vertices should transform an equal-area polygon with general $\mu_{i}$ into one with each $\mu_{i}=+1$. We gave such an example in the previous section, but it depended on the high symmetry of the $N=6$ case and we have been unable to generalize it. Also there may exist transformations in which two consecutive sides of an equal-area polygon are replaced by a single one, with other sides adjusted accordingly.

\section{REFERENCES}

1. I. J. Schoenberg, The finite Fourier series and elementary geometry, this MonTHLY 57 (1950) 390-404.

2. E. R. Berlekamp, E. N. Gilbert, and F. W. Snider, A polygon problem, this Monthly 72 (1965) 233-241.

3. H. S. M. Coxeter, Introduction to Geometry, 2nd. ed., John Wiley \& Sons, New York, 1969.

4. P. J. Davis, Circulant Matrices, John Wiley \& Sons, New York, 1979.

5. J. C. Fisher, D. Ruoff, and J. Shilletto, Polygons and polynomials, in The Geometric Vein: The Coxeter Festschrift, Springer-Verlag, New York, 1981, pp. 321-333. 
6. D. DeTemple and M. Hudelson, Square-banded polygons and affine regularity, this MonTHLY 108 (2001) 100-114.

7. S. Shteingold, One solution of the pentagon problem, J. Math. Behavior 13 (1994) 299-302.

8. B. Greer, Solving the pentagon problem, J. Math. Behavior 15 (1996) 151-154.

9. W. E. Ferguson, Jr., The construction of Jacobi and periodic Jacobi matrices with prescribed spectra, Math. Comp. 35 (1980) 1203-1220.

10. S. A. Andrea and T. G. Berry, Continued fractions and periodic Jacobi matrices, Linear Algebra Appl. 161 (1992) 117-134.

11. T. Muir, The Theory of Determinants in the Historical Order of Development, Dover, New York, 1960.

12. C. Brezinski, History of Continued Fractions and Padé Approximants, Springer-Verlag, Berlin, 1991.

13. H. M. Stark, An Introduction to Number Theory, MIT Press, Cambridge, 1978.

GUERSHON HAREL is a mathematics educator, whose research centers on cognition and epistemology of mathematics and their application in mathematics curricula and teacher education. His research includes the learning and teaching of undergraduate mathematics (such as linear algebra and proof) as well as elementary mathematics (such as fractions and linearity). Harel is currently a professor in the mathematics department at the University of California, San Diego. He has been a co-Director and co-Principal Investigator of the Algebraic Thinking Institute at UCSD, a professional development institute for algebra teachers, since 2000, when he moved from Purdue University to UCSD. Harel was born in Tunesia and entered high school in Israel, where he received his Ph.D. in mathematics from Ben-Gurion University in 1985. He enjoys sports, especially biking with his colleagues.

Dept. of Mathematics, University of California, San Diego, La Jolla, CA 92093

gharel@ucsd.edu

JEFFREY M. RABIN received a Ph.D. in physics from Stanford University in 1981. He is currently a professor of mathematics at UCSD, and the recipient of an Academic Senate Distinguished Teaching Award. In 1999 and 2000 he was co-Director of the Algebraic Thinking Institute at UCSD. His research interests include mathematical physics, algebraic geometry, and the geometry of supersymmetry. He enjoys playing traditional folk music on the hammered dulcimer.

Dept. of Mathematics, University of California, San Diego, La Jolla, CA 92093

jrabin@ucsd.edu 